\title{
Risperidone and clozapine were more cost effective than haloperidol and chlorpromazine in patients with schizophrenia
}

\author{
Glennie J. Technology overview: pharmaceuticals: pharmacoeconomic evaluations of clozapine in treatment-resistant \\ schizophrenia and risperidone in chronic schizophrenia. Ottawa (ON): Canadian Coordinating Office for Health Technology \\ Assessment (CCOHTA); $1997 \mathrm{Jul}$.
}

\section{Objective}

To compare the cost effectiveness and cost utilities of clozapine and risperidone with traditional therapy in patients with treatment resistant and chronic schizophrenia, respectively.

\section{Design}

Cost effectiveness and cost utility analyses from a government payer perspective using a decision model which incorporated meta-analyses, input from an expert panel to assess outcomes for each drug and its alternatives, and patient preferences derived from interviews with 7 patients who had schizophrenia. This overview reports the results of a study commissioned by CCHOTA to meet the above objective.*

\section{Setting}

Canada.

\section{Patients}

A theoretical cohort of patients in hospital who had treatment resistant schizophrenia was used in the model for evaluating clozapine. A second theoretical cohort of patients who had chronic schizophrenia was used to evaluate risperidone.

\section{Intervention}

In the model, each drug and its alternatives were assessed for the efficacy rate (success was defined as being well enough to be discharged), relapses, and the dropouts due to adverse effects and to lack of efficacy. The emergence of extrapyramidal symptoms was assessed for risperidone only. Clozapine was compared with haloperidol and chlorpromazine. Risperidone was compared with haloperidol, haloperidol decanoate, and fluphenazine decanoate.

\section{Main cost and outcome measures}

Cost saving per patient for 1 year, cost saving per patient over the patient's remaining lifetime (assumed to be $37 \mathrm{y}$ ), and the gain of quality adjusted life years (QALYs) for patients with schizophrenia. Only direct costs (1996 Canadian dollars) were included. Future costs and outcomes were discounted at a $5 \%$ rate.

\section{Main results}

In the clozapine analysis, clozapine was associated with the lowest cost and the highest number of QALYs. The cost saved per year for clozapine compared with chlorpromazine was $\$ 39000$ and 0.04 QALYs were gained. Over the patient's remaining lifetime, the cost saved was $\$ 682000$ and 0.70 QALYs were gained. In the risperidone analysis, risperidone was associated with the lowest cost and the highest number of QALYs. The cost saved per year for risperidone compared with haloperidol was $\$ 6500$ and 0.04 QALYs were gained. Over the patient's remaining lifetime, the cost saved was $\$ 114000$ and 0.70 QALYs were gained.

\section{Conclusions}

In Canada, using clozapine to treat patients who had treatment resistant schizophrenia was more cost effective than chlorpromazine or haloperidol. Using risperidone to treat patients with chronic schizophrenia was more cost effective than haloperidol, haloperidol decanoate, or fluphenazine decanoate.

*Oh P,Einarson TR, Iskedjian M, et al. Ottawa:CCOHTA, 1996.

Source of funding: The Canadian Coordinating Office for Health Technology Assessment (CCOHTA).

For article reprint:DrJ Glennie, c/o CCOHTA Publications, 110-955 Green Valley Crescent, Ottawa, Ontario K2C 3V4, Canada.Fax +16132265392;email pubs@ccohta.ca.

\section{Commentary}

This overview by Glennie of the CCOHTA study is a worthwhile addition to knowledge on cost effectiveness and one of the first cost utility analyses in schizophrenia.

The study modelled well defined outcomes of clozapine, $500 \mathrm{mg}$, in treatment resistant schizophrenia and risperidone, $6 \mathrm{mg}$, in chronic schizophrenia. Indirect social costs were not considered, but the findings remain relevant to psychiatric service providers. Drug effects were derived from evidence compatible with recent meta-analyses..$^{12}$ A sensitivity analysis varying the estimates of costs, outcomes, and utilities showed that the results were robust to clinically reasonable variations.

The cost effectiveness analysis suggests important annual and lifetime financial savings, mainly from reducing inpatient length of stay. The crucial rider is that the models depended on the availability of adequate community care, especially residential services. The annual cost utility increases were small (and without confidence intervals) but believable.

The results are also generalisable. The costs of schizophrenia, despite the fact that (or perhaps because) estimating them is an imprecise science, are as similar in westernised countries as the prevalence of the disorder. ${ }^{3}$

Ideally, we need evidence from "real life" controlled trials, or at least audit, that these savings will be made in everyday clinical practice. For example, financial gains cannot accrue in patients who cannot receive these treatments for clinical reasons such as non-compliance. More importantly, savings will only be made if the costs of inpatient care are reduced-that is, beds are closed. As most psychiatric services, at least in the UK, have found that bed numbers are already insufficient, the modelled cost effectiveness of atypical antipsychotic drugs appears impractical. These drugs benefit patients, but clinicians can only justify their use (to managers and the public) if they close beds. The only alternative is to spend more on the treatment of schizophrenia, but that is another matter.

\section{Stephen M Lawrie, MD University of Edinburgh Edinburgh, UK}

1 Wahlbeck K, Cheine M, Essali MA, et al. In: Cochrane Database of Systematic Reviews. The Cochrane Library. Oxford: Update Software, 1997 ; issue 4.

2 Kennedy E, Song F, Hunter R, et al. In: Cochrane Database of Systematic Reviews. The Cochrane Library. Oxford: Update Software, 1997; issue

3 Knapp M. Br J Psychiatry 1997;171:509-18. 